\title{
A Discrimination Theory Based on Categorical Variables and Its Application to Meterological Problems
}

\author{
By Eiichi Suzuki \\ Meteorological Research Institute, Tokyo \\ (Manuscript received 14 October 1968, in revised form 1 April 1969)
}

\begin{abstract}
The statistical discrimination theory was first proposed by C. R. Rao (1948) and has been widely applied to the several domains of scientific classification. Its theoretical development has been due to the work of C. R. Rao, S. S. Wilks, J. G. Bryan and others. It was recently applied to meteorological classifactory preciction by R. G. Miller (1962-1964) and by the present author (1963-1965) almost concurrently.

In this paper, a simple optimum linear discrimination function is firstly investigated for the case containing several qualitative or categorical variables only, based on the joint probability distribution (i.e., multi-variate binomial distribution) defined by A.S. Krishnamoorthy (1951) and by the author (1966).

Secondly the quantification procedure maximizing the correlation ratio between classified predictand variable $Y$ and categorical set of predictor variable $X$ used for classification (of $Y$ ), which has already been shown by C. Hayashi and S. Chino, is taken into account through combining it with a linear discriminant function.

Lastly, several explanatory examples are shown for qualitative prediction of the rain state and the occurrence or non-occurrence of snowslide by using the effective presaged predictors.
\end{abstract}

\section{Introduction}

Hitherto, the statistical linear discrimination function has been computed by using continuous stochastic variables only, and widely applied to scientific discriminatory problems. Some applications of it to meteorological prediction were recently attempted through technical contrivances by R. G. Miller and the author. However, in the case of mixed population (both discrete and continuous variables are to be taken into consideration at the same time) or of multi-variate binomial population, the problem of discrimination has not yet been studied, though a certain type of conditional probability has conventionally been used by the author.

The likelihood ratio based on multi-variate binomial distribution will be quite suggestive for discrimination fundamentally, as has been well known. Therefore, an approximately linearlized form of logarithmic likelihood ratio is induced for this case to serve the routine operation of forecasting in the sense of the least square procedure, and two special cases of the linearized discrimination index are practically applied in cate- gorical prediction of the local heavy rain state at the Hokuriku district in Japan.

In this approach, two states only of multiple presaged factors are to be taken into consideration since prediction of either of the two contingencies of the predictand is in question, but when several states or a more detailed classification of predictors has been actually given, the linear discrimination function is constituted through optimum quantifying categorical stochastic variables.

One optimum quantification has been proposed by C. Hayashi in direction of maximizing correlation ratio, instead of $\operatorname{tr}[$ (inverse matrix of covariance within) $\times$ (matrix of covariance between)].

After all, the ordinary linear discrimination function can be induced by this quantification and applied to discrimination concerning the occurrence or non-occurrence of snowslide by use of vegetational and geomorphological factors, and in this case, several originally quantitative variables (i. e., altitude and dimensions of the slope, etc.) are used by proper classifications because the population type containing categorical predictor variables only is the main object of this study. 


\section{Explicit form of likelihood ratio and its linea- rization in multi-variate binomial distribution}

Let $\left(X_{1}, X_{2}, \cdots \cdots, X_{S}\right)$ and $Y$ be s-variate discrimination predictor and a predictand respectively, and the following notations and assumptions are necessary to describe statistical relations:

$\pi_{i}(i=1,2)$ : population occurrence probability of a predictand $Y$ specified by

$$
P(Y \epsilon A)=\pi_{1}, \quad P(Y \epsilon B)=\pi_{2}, \pi_{1}+\pi_{2}=1
$$

where $\mathrm{A}$ and $\mathrm{B}$ are mutually exclusive and exhaustive cases of $\mathrm{Y}$.

$p_{i}, q_{i}(i=1,2, \cdots \cdots, s):$ population occurrence probability of predictor $X_{i}(i=1,2, \cdots, s)$ specified by

$$
P\left(X_{i}=1\right)=p_{i}, \quad P\left(X_{i}=0\right)=q_{i}=1-p_{i}
$$

$p_{A i}, \quad p_{B i}, q_{A i}, q_{B i}:$ conditional occurrence probability of predictor $X_{i}(i=1,2, \cdots, s)$ specified by

$$
\left.\begin{array}{ll}
P\left(X_{i}=1 / Y \epsilon A\right)=p_{A i}, & P\left(X_{i}=0 / Y \epsilon A\right)=q_{A i} \\
P\left(X_{i}=1 / Y \epsilon B\right)=p_{B i}, & P\left(X_{i}=0 / Y \epsilon B\right)=q_{B i}
\end{array}\right\}
$$

By these specifications, we have

$$
\left.\begin{array}{ll}
\left(p_{A i}+q_{A i}\right) \pi_{1}=\pi_{1}, & \left(p_{B i}+q_{B i}\right) \pi_{2}=\pi_{2} \\
p_{A i} \pi_{1}+p_{B i} \pi_{2}=p_{i}, & q_{A i} \pi_{1}+q_{B i} \pi_{2}=q_{i}
\end{array}\right\}
$$

and then, the corresponding relation between $X_{i}$ and $Y$ can be shown by the contingency Table 1 .

Table 1. Schematic contingency table showing the relation between $X_{i}$ and $Y$.

\begin{tabular}{c|c|c|c}
\hline$X_{i}$ & 1 & 0 & Total \\
\hline $\mathbf{A}$ & $p_{A i \pi_{1}}$ & $q_{A i} \pi_{1}$ & $\pi_{1}$ \\
$\mathbf{B}$ & $p_{B i \pi_{2}}$ & $q_{B i \pi_{2}}$ & $\pi_{2}$ \\
\hline Total & $p_{i}$ & $q_{i}$ & 1 \\
\hline
\end{tabular}

For the purpose of prediction for $Y$, we can assume the following inequality without loss of generality:

$$
p_{A i}>p_{B i}, \quad q_{A i}<q_{B i}(i=1,2, \cdots, s)
$$

since we can put $X_{i}$ as 1 when the state of $X_{i}$ is more favorable for the case $Y \epsilon A$ than for the other exclusive case $Y \epsilon B$.

Multi-variate binomial distribution was indepen dently investigated by A.S. Krishnamoorthy (1951) and E. Suzuki(1966) and could be expressed after all as follows:

$$
\begin{aligned}
F_{A}(\boldsymbol{X}) & \equiv P\left(X_{i}=x_{i}, \quad i=1,2, \cdots, s / A\right) \\
& =\prod_{i=1}^{s} p_{A i} \quad{ }^{x_{i}} \quad q_{A i} G_{A}(\boldsymbol{X})
\end{aligned}
$$

where

$$
\left.\begin{array}{c}
G_{A}(\boldsymbol{X})=1+\sum_{i<j}^{s} \rho_{i j(A)} \frac{\left(x_{i}-p_{A i}\right)\left(x_{j}-p_{A j}\right)}{\sqrt{p_{A i} q_{A i} p_{A j} q_{A j}}} \\
+\sum_{i<j<k}^{s} E\left\{\left(x_{i}-p_{A i}\right) \cdot\left(x_{j}-p_{A j}\right)\left(x_{k}-p_{A k}\right)\right\} \\
\frac{\left(x_{i}-p_{A i}\right)\left(x_{j}-p_{A j}\right)\left(x_{k}-p_{A k}\right)}{p_{A i} q_{A i} p_{A j} q_{A j} p_{A k} q_{A k}}+\cdots \cdots+ \\
E\left\{\prod_{i=1}^{s}\left(x_{i}-p_{A i}\right)\right\} \prod_{i=1}^{s}\left(x_{i}-p_{A i}\right) / \prod_{i=1}^{s} p_{A i} q_{A i} \\
\left(x_{i}, x_{j}, x_{k}=0 \text { or } 1, i=1,2, \cdots, s\right)
\end{array}\right\}
$$

where $G_{B}(\boldsymbol{X})$ has a similar form to $G_{A}(\boldsymbol{X})$ obtained by substituting subscript $B$ for subscript $A$ in (2.6), and $\rho_{i j}(A)$ is the correlation coefficient between $X_{i}$ and $X_{j}$ in the case $Y \epsilon A$, and its estimator is evidently equivalent to the coefficient of association.

In order to induce the discriminator for $\mathrm{Y}$, the next condition must be considered fundamentally:

$$
\frac{P_{A}(\boldsymbol{X})}{P_{B}(\boldsymbol{X})} \gtrless 1 \text { or } \log \frac{P_{A}(\boldsymbol{X})}{P_{B}(\boldsymbol{X})} \gtrless 0,
$$

that is to say, if $\mathrm{I}=\log \left\{P_{A}(\boldsymbol{X}) / P_{B}(\boldsymbol{X})\right\}$ is positive for the actual data set $\boldsymbol{X}=\left(X_{1}, X_{2}, \cdots, X_{s}\right)$, $Y$ can be regarded as belonging to $A$ and if $I$ is negative, the reverse action will be taken up on the contrary.

The explicit form of discriminator $I$ can be written by

$$
\begin{aligned}
I= & \sum_{i=1}^{s} \log \left(q_{A i} / q_{B i}\right)+\sum_{i=1}^{s} x_{i} \log \left(p_{A i} q_{B i} / p_{B i} q_{A i}\right) \\
& +\log \left\{G_{A}(\boldsymbol{X}) / G_{B}(\boldsymbol{X})\right\}
\end{aligned}
$$

and we can discriminate for category $A$ or $B$ by use of the sample estimate of $I$.

However, the actual computation of $I$ is rather difficult since the 3 rd term of the right-hand side of (2.8) can hardly be computed without the help of the electronic computer's routine work except for the case of small $s$, even if all the parameters were readily estimated by a given sample set. Also, the estimation procedures of sample error variances of parameters were never obtained easily.

For the case when $s$ is equal to 2 and 3 only, 
the actual examples of computation of (2.8) will be shown later.

To serve for the operational routine of prediction, we may consider the best linear form of discriminator $I$ as follows:

If we put

$$
\begin{aligned}
& f\left(x_{1}, x_{2}, \cdots, x_{s}\right) \equiv \log \left\{G_{A}(X) / G_{B}(X)\right\} \\
& =\log \left[1+\sum_{i<j}^{s} \rho_{i j}(A) \frac{\left(x_{i}-p_{A i}\right)\left(x_{j}-p_{A j}\right)}{\sqrt{p_{A i} q_{A i} p_{A j} q_{A j}}}+\cdots\right. \\
& \left.+E\left\{\prod_{i=1}^{s}\left(x_{i}-p_{A i}\right)\right\} \prod_{i=1}^{s} \frac{\left(x_{i}-p_{A i}\right)}{p_{A i} q_{A i}}\right]-\log [1+ \\
& \sum_{i<j}^{s} \rho_{i j(B)} \frac{\left(x_{i}-p_{B i}\right)\left(x_{j}-p_{B j}\right)}{\sqrt{p_{B i} q_{B i} p_{B j} q_{B j}}}+\cdots \\
& \left.+E\left\{\prod_{i=1}^{s}\left(x_{i}-p_{B i}\right)\right\} \prod_{i=1}^{s} \frac{\left(x_{i}-p_{B i}\right)}{p_{B i} q_{B i}}\right]
\end{aligned}
$$

then the linear approximation of $f(\cdots)$ can be written by

$$
f\left(x_{1}, x_{2}, \cdots, x_{s}\right)=\sum_{i=1}^{s} a_{i} x_{i}+\varepsilon\left(x_{1}, x_{2}, \cdots, x_{s}\right)
$$

and the next two methods will be considered:

Least square method:

$$
\sum_{x_{1}=0}^{1} \sum_{x_{2}=0}^{1} \cdots \sum_{x_{s}=0}^{1} \varepsilon\left(x_{1}, x_{2}, \cdots, x_{s}\right)^{2} \rightarrow \text { Min. }
$$

Method of mini-max approximation:

$$
\underset{x_{1}, x_{2}, \cdots, x_{s}}{\operatorname{Max} .}\left|\varepsilon\left(x_{1}, x_{2}, \cdots, x_{s}\right)\right| \rightarrow \text { Min. }
$$

Since the former method is used in general, almost equal results are expected from these two methods except for cases where extremes values are taken by parameters $p_{A i}, q_{A i}, p_{B i}, q_{B i}$ and $\rho_{i j}$ etc. (The telescoping method due to $C$. Lanczos is quite suggestive for the latter method but its computational procedure is rather difficult in this case).

Then, we have the following theorem:

\section{[Theorem]}

Let the best linear form of $I$ be

$I=$ const. $+\sum_{i=1}^{s} w_{i} X_{i}$ in the sense of the least square error, then we have

$$
\begin{aligned}
& \text { const. }=\sum_{i=1}^{s} \log \left(q_{A i} / q_{B i}\right) \quad w_{i}=\log \left(p_{A i} q_{B i} / p_{B i} q_{A i}\right) \\
& +2^{2-s}\left\{g_{i}-\sum_{i=1}^{s} g_{i} /(s+1)\right\}
\end{aligned}
$$

and the minimum sum of square error for this linear approximation can be shown by

$$
\begin{aligned}
& \text { Min. } S=\sum_{x_{1}=0}^{1} \sum_{x_{2}=0}^{1} \ldots \sum_{x s=0}^{1}\left[\log \left\{G_{A}(\boldsymbol{X}) / G_{B}(\boldsymbol{X})\right\}\right]^{2} \\
& +2^{2-s}\left\{\left(\sum_{i=1}^{s} g_{i}\right)^{2} /(s+1)-\sum_{i=1}^{s} g_{i}{ }^{2}\right\}
\end{aligned}
$$

where

$$
g_{i}=\sum_{x_{k}} \sum_{\left(\neq x_{i}\right)} \cdots\left[\log \left\{G_{A}(\boldsymbol{X}) / G_{B}(\boldsymbol{X})\right\}\right]_{x_{i}=1}
$$

Proof of this theorem is shown in the Appendix. And so the mean standard error of linearization can be tentatively written by

$$
\sqrt{\operatorname{Min} . S / 2^{s}}
$$

To have a clarified example, we may consider two special cases $s=2$ and $s=3$, in connection with the actual computations to be shown later.

Case. 1. $s=2$.

$$
\begin{aligned}
& f\left(x_{1}, x_{2}\right)=\log \left\{1+\rho_{12(A)} \frac{\left(x_{1}-p_{A_{1}}\right)\left(x_{2}-p_{A_{2}}\right)}{\sqrt{\prime} p_{A_{1}} q_{A_{1}} p_{A_{2}} q_{A_{2}}}\right\} \\
& -\log \left\{1+\rho_{12(B)} \frac{\left(x_{1}-p_{B_{1}}\right)\left(x_{2}-p_{B_{2}}\right)}{\sqrt{p_{B_{1}} q_{B_{1}} p_{B_{2}} q_{B_{2}}}}\right\} \\
& S\left(a_{1}, a_{2}\right)=f(0,0)^{2}+\left\{f(1,0)-a_{1}\right\}^{2} \\
& +\left\{f(0,1)-a_{2}\right\}^{2}+\left\{f(1,1)-a_{1}-a_{2}\right\}^{2} \\
& \partial S / \partial a_{1}=0 \rightarrow 2 a_{1}+a_{2}=f(1,0)+f(1,1) \\
& \partial S / \partial a_{2}=0 \rightarrow a_{1}+2 a_{2}=f(0,1)+f(1,1) \\
& a_{1}=\frac{2 f(1,0)-f(0,1)+f(1,1)}{3} \\
& a_{2}=\frac{2 f(0,1)-f(1,0)+f(1,1)}{3}
\end{aligned}
$$

Min. $S=f(0,0)^{2}+\{f(1,0)+f(0,1)$

$$
-f(1,1)\}^{2} / 3
$$

Case 2. $s=3$.

$$
\begin{gathered}
f\left(x_{1}, x_{2}, x_{3}\right)=\log \left\{1+\rho_{12(A)} \frac{\left(x_{1}-p_{A_{1}}\right)\left(x_{2}-p_{A_{2}}\right)}{\sqrt{p_{A_{1}} q_{A_{1}} p_{A_{2}} q_{A_{2}}}}+\rho_{13(A)} \frac{\left(x_{1}-p_{A_{1}}\right)\left(x_{3}-p_{A_{3}}\right)}{\sqrt{p_{A_{1}} q_{A_{1}} p_{A_{3}} q_{A_{3}}}}\right. \\
\left.+\rho_{23(A)} \frac{\left(x_{2}-p_{A_{2}}\right)\left(x_{3}-p_{A_{3}}\right)}{\sqrt{p_{A_{2}} q_{A_{2}} p_{A_{3}} q_{A_{3}}}}+\rho_{123(A)} \frac{\left(x_{1}-p_{A_{1}}\right)\left(x_{2}-p_{A_{2}}\right)\left(x_{3}-p_{A_{3}}\right)}{\sqrt{p_{A_{1}} q_{A_{1}} p_{A_{2}} q_{A_{2}} p_{A_{3}} q_{A_{3}}}}\right\} \\
-\log \left\{1+\rho_{12(B)} \frac{\left(x_{1}-p_{B_{1}}\right)\left(x_{2}-p_{B_{2}}\right)}{\sqrt{p_{B_{1}} q_{B_{1}} p_{B_{2}} q_{B_{2}}}}+\rho_{13(B)} \frac{\left(x_{1}-p_{B_{1}}\right)\left(x_{3}-p_{B_{3}}\right)}{\sqrt{p_{B_{1}} q_{B_{1}} p_{B_{3}} q_{B_{3}}}}\right.
\end{gathered}
$$




$$
\left.\begin{array}{l}
\left.\quad+\rho_{23(B)} \frac{\left(x_{2}-p_{B_{2}}\right)\left(x_{3}-p_{B_{3}}\right)}{\sqrt{p_{B_{2}} q_{B_{2}} p_{B_{3}} q_{B_{3}}}}+\rho_{123(B)} \frac{\left(x_{1}-p_{B_{1}}\right)\left(x_{2}-p_{B_{2}}\right)\left(x_{3}-p_{B_{3}}\right)}{\sqrt{p_{B_{1}} q_{B_{1}} p_{B_{2}} q_{B_{2}} p_{B_{3}} q_{B_{3}}}}\right\} \\
\begin{array}{l}
S\left(a_{1}, a_{2}, a_{3}\right)=f(0,0,0)^{2}+\left\{f(1,0,0)-a_{1}\right\}^{2}+\left\{f(0,1,0)-a_{2}\right\}^{2}+\left\{f(0,0,1)-a_{3}\right\}^{2} \\
\quad+\left\{f(1,1,0)-a_{1}-a_{2}\right\}^{2}+\left\{f(1,0,1)-a_{1}-a_{3}\right\}^{2}+\left\{f(0,1,1)-a_{2}-a_{3}\right\}^{2} \\
\quad+f\left\{(1,1,1)-a_{1}-a_{2}-a_{3}\right\}^{2}
\end{array} \\
\left.\begin{array}{l}
4 a_{1}+2\left(a_{2}+a_{3}\right)=f(1,0,0)+f(1,1,0)+f(1,0,1)+f(1,1,1) \\
4 a_{2}+2\left(a_{1}+a_{3}\right)=f(0,1,0)+f(0,1,1)+f(1,1,0)+f(1,1,1)
\end{array}\right\} \\
4 a_{3}+2\left(a_{1}+a_{2}\right)=f(0,0,1)+f(1,0,1)+f(0,1,1)+f(1,1,1)
\end{array}\right\}
$$

\section{Quantification for qualitative predictors and linear discriminator.}

The ordinary linear discrimination function has been computed by using continuous variables only, and not yet given in the case when qualitative or discrete variables are to be taken into account.

However, in such a case, discriminant analysis will be carried out through a certain suitable quantification for these variables as will be stated below, since the linear discriminant function can be constituted by considering these quantified variables as continuous variables tentatively.

Now, let us take up the case where all predictors $X_{1}, X_{2}, \cdots, X_{s}$ are categorical variables only and consider an optimum quantification for these variables.

The actual procedure is schematically shown in Table 2, to explain the quantification process.

In Table 2, let the category size of each variable be $k_{1}, k_{2}, \cdots, k_{s}$ and $\left(x_{11}, \cdots, x_{1 k_{1}}\right),\left(x_{21}, \cdots, x_{2 k_{2}}\right)$ $\cdots,\left(x_{s 1}, \cdots, x_{s k s}\right)$ be the sets of quantification result for category sets $\left(C_{11}, \cdots, C_{1 k_{1}}\right),\left(C_{21}, \cdots\right.$, $\left.C_{2 k 2}\right), \cdots,\left(C_{s 1}, \cdots C_{s k s}\right)$ respectively, then the total sample size $\mathrm{n}$ is divided into $n_{A}$ (sample size in the case $Y \epsilon A$ and $n_{B}$ (sample size in the case $Y \epsilon B$ ) and check mark $\vee$ can be assigned to any one category of each predictor by the dependent data.

If quantification is carried out by an appropriate method, their totals will be attainable as shown in Table 2.

Therefore, we put each mean value and variance as

$$
\begin{gathered}
\bar{X}_{A i}=\sum_{j} X_{i j A} / n_{A}, \quad \bar{X}_{B i}=\sum_{j} X_{i j B} / n_{B} \\
s^{2}{ }_{A i}=\sum_{j}\left(X_{i j A}-\bar{X}_{A i}\right)^{2} / n_{A}, \\
s^{2}{ }_{B j}=\sum_{j}\left(X_{i j B}-\bar{X}_{B i}\right)^{2} / n_{B} \\
(i=1,2, \cdots, s)
\end{gathered}
$$

then we have squared mean distance relative to variance within two cases as shown by the next formula.

$$
\eta^{2}{ }_{A B}(i)=n_{A} n_{B}\left(\bar{X}_{A i}-\bar{X}_{B i}\right)^{2} /\left(n_{A} s^{2}{ }_{A i}+n_{B} s^{2}{ }_{B i}\right)
$$

and $\gamma^{2}{ }_{A B}(i)(i=1,2, \cdots, s)$ is essentially proportional to the correlation ratio between the two cases A and B for a certain predictor $X_{i}$.

To attain the optimum quantification in a statistical sense, two approaches at least can be considered as follows:

(i) If we put $W_{s}, \boldsymbol{B}_{s}$ as $\mathrm{s} \times \mathrm{s}$ variance-covariance matrix of predictors $X_{1}, X_{2}, \cdots, X_{s}$ within two cases $Y \epsilon A$ and $Y \epsilon B$, and between these two cases respectively, as has often been used in the field of Statistical Analysis of Variance, then $\operatorname{tr}\left(W_{s}{ }^{-1}\right.$ $\boldsymbol{B}_{s}$ ) is a fundamentally proportional quantity to the generalized relative squared distance defined by P. C. Mahalanobis already, so the first approach is to maximize $\operatorname{tr}\left(\boldsymbol{W}^{-1}\right.$ s. $\left.\boldsymbol{B}_{s}\right)$ in regard to $x_{i j}$.

(ii) The second approach is to maximize $\eta^{2}{ }_{A B}(i)$ in regard to each $X_{i j}(i=1,2, \cdots, s)$. Statistical dependency or relation between $X_{i}$ and $X_{j}$ is taken into consideration in the first approach but not in the second one; so, if possible, (i) is more preferable than (ii). 
Table 2. Schematic illustration of actual summarizing process of dependent samples.

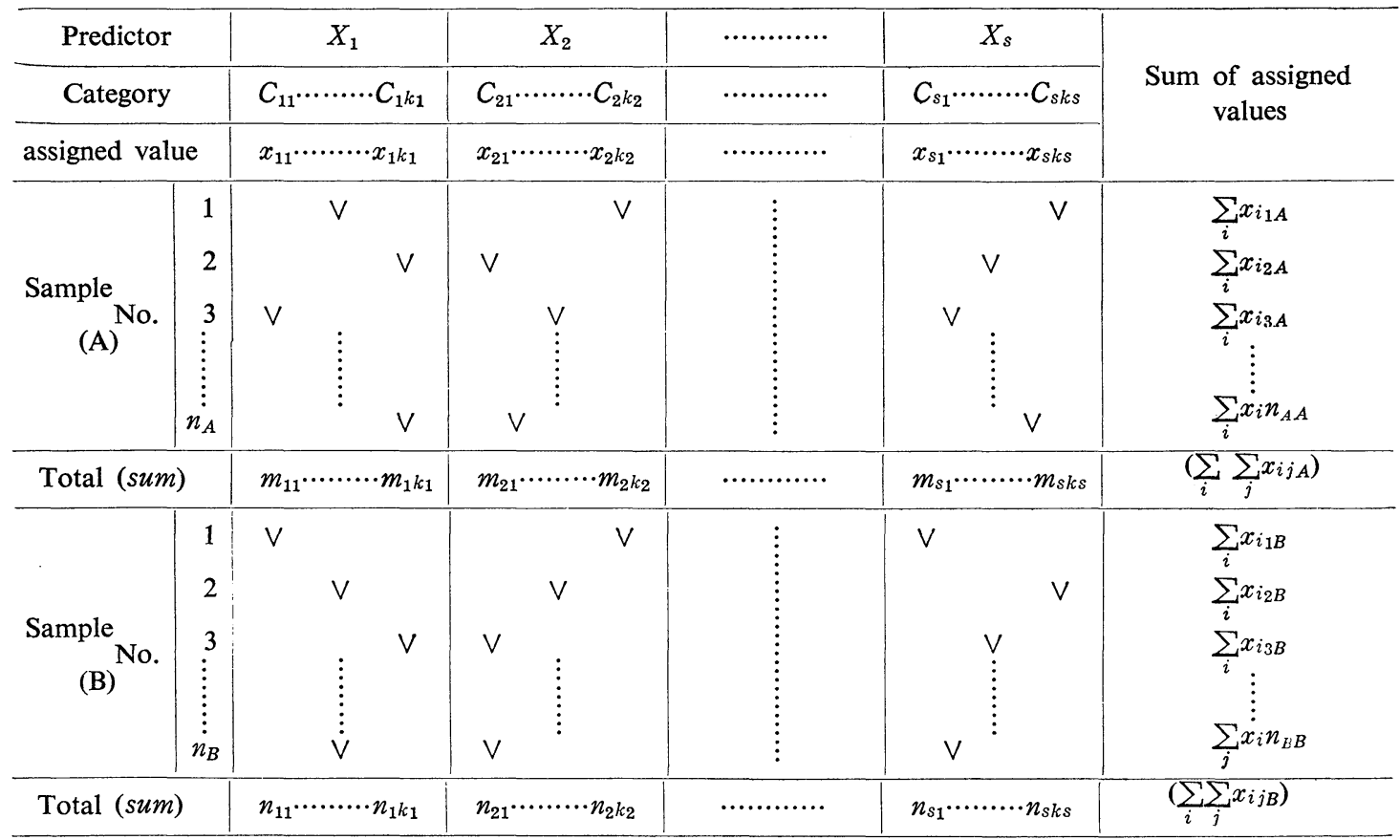

Remark: $\sum_{i} x_{i j_{A}}\left(j=1, \cdots, n_{A}\right)$ and $\sum_{i} x_{i j_{B}}\left(j=1, \cdots, n_{B}\right)$ show the summations of assigned values in the columns for the cases of $\mathrm{A}$ and $\mathrm{B}$ respectively.

However, an analytical procedure to maximize $\operatorname{tr}\left(\boldsymbol{W}^{-1} \boldsymbol{B}\right)$ in respect to all variables $x_{i j}(j=1$, $\left.2, \cdots, k_{i}, i=1,2, \cdots, s\right)$ is very difficult to attain and since results essentially equivalent to those by the first approach are expected at least from constituting an ordinary linear discriminant function after quantification for each predictor $X_{i}$ by use of the second approach we will make use of this latter.

The following results have already been reported by C. Hayashi (1952) and S. Chino (1963), to maximize $\eta^{2}{ }_{A B}(i)$ in respect to $x_{i j}$ :

$$
\frac{\partial \eta_{A}^{2}{ }_{B}(i)}{\partial x_{i j}}=0 \quad\left(j=1,2, \cdots, k_{i}\right)
$$

condition 1. $\bar{X}_{i}=\left(n_{A} \bar{X}_{A i}+n_{B} \bar{X}_{B i}\right) /\left(n_{A}+n_{B}\right)=0$ condition 2. $\sum_{j=1}^{k i} m_{i j}=n_{A}, \sum_{j=1}^{k i} n_{i j}=n_{B}$

solution:

$$
\begin{aligned}
& x_{i j}=C\left(\frac{m_{i j}}{n_{A}}-\frac{n_{i j}}{n_{B}}\right) /\left(m_{i j}+n_{i j}\right) \\
& \left(j=1,2, \cdots, k_{i}, \quad i=1,2, \cdots, s\right)
\end{aligned}
$$

where $\mathrm{C}=$ arbitrary const. and, $m_{i j} n_{i j}$ are such numbers as given in Table 2.

After quantification for each category by using the above solution, we can compute sample variance $\operatorname{Var}\left(X_{i}\right)$ and sample covariance Cov. $\left(X_{i}\right.$, $X_{j}$ ) as follows:

$$
\begin{gathered}
\operatorname{Var}\left(X_{i}\right)=\left\{\sum_{j}\left(X_{i j A}-\bar{X}_{i A}\right)^{2}\right. \\
\left.+\sum_{j}\left(X_{i j B}-\bar{X}_{i B}\right)^{2}\right\} /\left(n_{A}+n_{B}\right) \equiv V_{i i} \\
\operatorname{Cov} .\left(X_{i}, X_{j}\right)=\left\{\sum_{k}\left(X_{i k A}-\bar{X}_{i A}\right)\left(X_{j k A}-\bar{X}_{j A}\right)\right. \\
\left.+\sum_{k}\left(X_{i k B}-\bar{X}_{i B}\right) \times\left(X_{j k B}-\bar{X}_{j B}\right)\right\} / \\
\left(n_{A}+n_{B}\right) \equiv V_{i j} \quad(i, j=1,2, \cdots, s)
\end{gathered}
$$

And if we let the linear discriminant function be

$$
I=w_{1} X_{1}+w_{2} X_{2}+\cdots+w_{s} X_{s}
$$

as in the case containing continuous variables only, then the coefficient vector $\left(w_{1}, w_{2}, \cdots, w_{s}\right)$ can be obtained by solving the next equation: 


$$
\left(\begin{array}{cccc}
V_{11} & V_{12} & \cdots & V_{1 s} \\
V_{21} & V_{22} & \cdots & V_{2 s} \\
\cdots \cdots \cdots \cdots \cdots \cdots & \cdots \cdots \\
V_{s 1} & V_{s 2} & \cdots & V_{s s}
\end{array}\right)\left(\begin{array}{c}
w_{1} \\
w_{2} \\
\vdots \\
w_{s}
\end{array}\right)=\lambda\left(\begin{array}{c}
\bar{X}_{A_{1}}-\bar{X}_{B_{1}} \\
\bar{X}_{A 2}-\bar{X}_{B_{2}} \\
\vdots \\
\bar{X}_{A s}-\bar{X}_{B s}
\end{array}\right)
$$

where $\lambda$ is an arbitrary constant.

To select effective predictors in discrimination, the following procedure has been proposed by R. G. Miller (1962),

The first predictor $X_{(1)}$ must satisfy the condition Mix. $\gamma^{2}{ }_{A B}(i)$, and the successive effective predictors must satisfy the condition $\operatorname{Max} \operatorname{tr}\left(W^{-1}\right.$ $\boldsymbol{B})$ sequentially under the assumption that both $\boldsymbol{W}$ and $\boldsymbol{B}$ are computed only by taking up the predictors adopted in each step of this sequential process.

The above-mentioned procedures can also be applid to the case of quantified predictor variables as will be mentioned below.

\section{Application Examples of the theoretical results obtained above}

(i) Discrimination between heavy rainfall and not heavy rainfall (including no rainfall) based on the state of the low level Jet.

The June-August data, which are necessary for predicting the rainfall state at the Hokuriku district, have been obtained for the period 1956 -1959 by S. Miyazawa and I. Maeda, and the next notaions and assumptions are adopted here: Event $Y \epsilon A=$ Daily maximum rainfall is equal to or greater than $100 \mathrm{~mm}$ at any station in the Hokuriku district.

Event $Y \epsilon B=$ Any possible cases other than $Y \epsilon A$. $X_{1}=$ Variable is equal to 1 or 0 corresponding to the existence or nonexistence of the low-level Jet at Wajima respectively.

(This existence can be recognized by a conspicuous maximum wind velocity in the lower wind profile) $X_{2}=$ Similar variable to above at Yonago. $X_{3}=$ Variable is equal to 1 or 0 corresponding to the case that the mean Showalter's stability index is less than or equal to $2^{\circ} \mathrm{C}$ in Akita, Yonago and Wajima. Variable showing the mean values of Showalter's Stability index may be obtained by a similar process even when we make use of any other three points.

Summarized contingency tables are shown in Table 3 , to show the various corresponding relations among $Y, X_{1}, X_{2}$ and $X_{3}$, and sample estimates of parameters are computed by using Table 3 and shown in Table 4.

Computation results are obtained for example as follows:

$$
\begin{aligned}
& \hat{\pi}_{1}=\frac{n_{A}}{n}=0.417 \quad \hat{\pi}_{2}=1-\hat{\pi}_{1}=0.583 \\
& \hat{p}_{A_{1}}=32 / 50=0.640 \quad \hat{q}_{A_{1}}=1-\hat{p}_{A_{1}}=0.360 \\
& \hat{p}_{B_{1}}=29 / 70=0.414 \quad \hat{q}_{B_{1}}=1-\hat{p}_{B_{1}}=0.586 \\
& \tilde{\rho}\left(Y, X_{1}\right)=\frac{18 \times 29-41 \times 32}{\sqrt{59 \times 61 \times 50 \times 70}}=-0.223 \\
& \tilde{\rho}\left(X_{1}, X_{2}\right)=\frac{34 \times 39-25 \times 22}{\sqrt{59 \times 61 \times 56 \times 64}}=0.216 \\
& \tilde{\rho}_{12(A)}=\frac{14 \times 21-7 \times 8}{\sqrt{21 \times 29 \times 22 \times 28}}=0.389 \\
& \tilde{\rho}_{12(B)}=\frac{23 \times 21-11 \times 15}{\sqrt{38 \times 32 \times 34 \times 36}}=0.261
\end{aligned}
$$

(a) Case 1. $s=2$

To predict discriminately the case $\mathrm{A}$ or the case B of $\mathrm{Y}$ by use of two predictors $X_{1}$ and $X_{2}$, it is necessary to obtain the discriminator $I$ by the following computations:

$$
\text { const. }=\log \left(\hat{q}_{A_{1}} / \hat{q}_{B_{1}}\right)+\log \left(\hat{q}_{A_{2}} / \hat{q}_{B_{2}}\right)=-1.0666
$$

Table 3. Contingency tables showing corresponding relations among $Y, X_{1}, X_{2}$ and $X_{3}$.

\begin{tabular}{c|c|c|c}
\hline$X_{1}$ & 0 & 1 & Total \\
\hline $\mathbf{A}$ & 18 & 32 & 50 \\
$\mathbf{B}$ & 41 & 29 & 70 \\
\hline Total & 59 & 61 & 120 \\
\hline
\end{tabular}

\begin{tabular}{c|c|c|c}
\hline$X_{2}$ & 0 & 1 & Total \\
\hline A & 16 & 34 & 50 \\
B & 40 & 30 & 70 \\
\hline Total & 56 & 64 & 120 \\
\hline
\end{tabular}

\begin{tabular}{c|c|c|c}
\hline$X_{3}$ & 0 & 1 & Total \\
\hline A & 21 & 29 & 50 \\
B & 50 & 20 & 70 \\
\hline Total & 71 & 49 & 120 \\
\hline
\end{tabular}




\begin{tabular}{c|c|c|c}
\hline$X_{2}$ & 0 & 1 & Total \\
\hline 0 & 34 & 22 & 56 \\
1 & 25 & 39 & 64 \\
\hline Total & 59 & 61 & 120 \\
\hline
\end{tabular}

\begin{tabular}{c|c|c|c}
\hline$X_{3}$ & 0 & 1 & Total \\
\hline 0 & 40 & 31 & 71 \\
1 & 19 & 30 & 49 \\
\hline Total & 59 & 61 & 120 \\
\hline
\end{tabular}

\begin{tabular}{c|c|c|c}
\hline$X_{3} X_{2}$ & 0 & 1 & Total \\
\hline 0 & 38 & 33 & 71 \\
1 & 18 & 31 & 49 \\
\hline Total & 56 & 64 & 120 \\
\hline
\end{tabular}

Case $Y \in A$

\begin{tabular}{c|r|r|c}
\hline$X_{2}$ & 0 & 1 & Total \\
\hline 0 & 14 & 8 & 22 \\
1 & 7 & 21 & 28 \\
\hline Total & 21 & 29 & 50 \\
\hline
\end{tabular}

\begin{tabular}{c|r|r|r}
\hline$X_{1}$ & 0 & 1 & Total \\
\hline 0 & 17 & 21 & 38 \\
1 & 4 & 8 & 12 \\
\hline Total & 21 & 29 & 50 \\
\hline
\end{tabular}

\begin{tabular}{c|r|r|c}
\hline$X_{3} X_{2}$ & 0 & 1 & Total \\
\hline 0 & 16 & 22 & 38 \\
1 & 6 & 6 & 12 \\
\hline Total & 22 & 28 & 50 \\
\hline
\end{tabular}

\section{Case $Y \in B$}

\begin{tabular}{c|c|c|c}
\hline$X_{2}$ & 0 & 1 & Total \\
\hline 0 & 23 & 11 & 34 \\
1 & 15 & 21 & 36 \\
\hline Total & 38 & 32 & 70 \\
\hline
\end{tabular}

\begin{tabular}{c|c|c|c}
$X_{3} X_{1}$ & 0 & 1 & Total \\
\hline 0 & 21 & 12 & 33 \\
1 & 17 & 20 & 37 \\
\hline Total & 38 & 32 & 70 \\
\hline
\end{tabular}

\begin{tabular}{c|c|c|c}
\hline$X_{3} \bar{X}_{2}$ & 0 & 1 & Total \\
\hline 0 & 19 & 14 & 33 \\
1 & 15 & 22 & 37 \\
\hline Total & 34 & 36 & 70 \\
\hline
\end{tabular}

Table 4. Statistical quantities and estimates of parameters

\begin{tabular}{c|c|c|c|c}
\hline$n$ & $n_{A}$ & $n_{B}$ & $\widehat{\pi}_{1}$ & $\widehat{\pi}_{2}$ \\
\hline 120 & 50 & 70 & 0.417 & 0.583 \\
\hline
\end{tabular}

\begin{tabular}{l|l|l|l|l|l}
\hline$i$ & $\widehat{p}_{A i}$ & $\widehat{q}_{A i}$ & $\widehat{p}_{B i}$ & $\widehat{q}_{B i}$ & $\tilde{\rho}\left(Y, X_{i}\right)$ \\
\hline 1 & 0.640 & 0.360 & 0.414 & 0.586 & -0.2226 \\
2 & 0.680 & 0.320 & 0.429 & 0.571 & -0.2485 \\
3 & 0.580 & 0.420 & 0.286 & 0.714 & -0.2952 \\
\hline
\end{tabular}

\begin{tabular}{c|c|c|c|c|c|c|c|c|c}
\hline & \multicolumn{3}{|c|}{$\tilde{\rho}\left(X_{i}, X_{j}\right)$} & \multicolumn{3}{c|}{$\tilde{\rho}_{i j(A)}$} & \multicolumn{3}{c}{$\tilde{\rho}_{i j(B)}$} \\
\hline$i$ & 1 & 2 & 3 & 1 & 2 & 3 & 1 & 2 & 3 \\
\hline 1 & 1.0000 & 0.2161 & 0.1727 & 1.0000 & 0.3886 & 0.0987 & 1.0000 & 0.2607 & 0.1773 \\
2 & & 1.0000 & 0.1654 & & 1.0000 & 0.0038 & & 1.0000 & 0.1701 \\
3 & & & 1.0000 & & & & 1.0000 & & 1.0000 \\
\hline
\end{tabular}

Remark:

$n$ : total sample size

$\hat{p}_{A i}, \hat{q}_{A i}$, etc. : sample estimates of probability

$\tilde{\rho}()$ etc. : sample correlation coefficients between two variables shown in brackets

$$
f(1,1)=\log \left\{1+\tilde{\rho}_{12(A)} \sqrt{\frac{\hat{q}_{A_{1}} \hat{q}_{A 2}}{\hat{p}_{A_{1}} \hat{p}_{A_{2}}}}\right\}-\log \left\{1+\tilde{\rho}_{12(B)} \sqrt{\frac{\hat{q}_{B_{1}} \hat{q}_{B_{2}}}{\hat{p}_{B_{1}} \hat{p}_{B_{2}}}}\right\}=-0.1237
$$




$$
\begin{aligned}
& f(1,0)=\log \left\{1-\tilde{\rho}_{12(A)} \sqrt{\frac{\hat{q}_{A 1} \hat{p}_{A 2}}{\hat{p}_{A_{1}} \hat{q}_{A_{2}}}}\right\} \\
& -\log \left\{1-\tilde{\rho}_{12(B)} \sqrt{\frac{\hat{q}_{B_{1}} \hat{p}_{B_{2}}}{\hat{p}_{B_{1}} \hat{q}_{B_{2}}}}\right\}=-0.2405 \\
& f(0,1)=\log \left\{1-\tilde{\rho}_{12(A)} \sqrt{\frac{\hat{p}_{A 1} \hat{q}_{A_{2}}}{\hat{q}_{A_{1}} \hat{p}_{A_{2}}}}\right\} \\
& -\log \left\{1-\tilde{\rho}_{12(B)} \sqrt{\frac{\hat{p}_{B_{1}} \hat{q}_{B_{2}}}{\hat{q}_{B_{1}} \hat{p}_{B 2}}}\right\}=-0.1473 \\
& f(0,0)=\log \left\{1+\tilde{\rho}_{12(A)} \sqrt{\frac{\hat{p}_{A 1} \hat{p}_{A 2}}{\hat{q}_{A_{1}} \hat{q}_{A_{2}}}}\right\} \\
& -\log \left\{1+\tilde{\rho}_{12(B)} \sqrt{\frac{\hat{p}_{B_{1}} \hat{p}_{B_{2}}}{\hat{q}_{B_{1}} \hat{q}_{B_{2}}}}\right\}=0.3888 \\
& a_{1}=\frac{2 f(1,0)-f(0,1)+f(1,1)}{3}=-0.1525 \\
& a_{2}=2 f(0,1)-\frac{f(1,0)+f(1,1)}{3}=-0.0592 \\
& w_{1}=\log \left(\hat{p}_{A_{1}} \hat{q}_{B_{1}} / \hat{p}_{B_{1}} \hat{q}_{A_{1}}\right)+a_{1}=0.7691 \\
& w_{2}=\log \left(\hat{p}_{A_{2}} \hat{q}_{B_{2}} / \hat{p}_{B_{2}} \hat{q}_{A_{2}}\right)+a_{2}=0.9822 \\
& \text { Min. } S=f(0,0)^{2}+\frac{\{f(1,0)+f(0,1)-f(1,1)\}^{2}}{3} \\
& =0.033
\end{aligned}
$$

To verify the prediction accuracy of the exact discriminator I defined by (2.8) and of the approximate linear discriminator for the dependent data, the correspondence between discriminated results and actual cases is given by Table 5 .

Sample means $\bar{I}_{A}$ and $\bar{I}_{B}$ are obtained by

$$
\begin{aligned}
& \bar{I}_{A}=\text { Const. }+w_{1} \times \hat{p}_{A_{1}}+w_{2} \times \hat{p}_{A_{2}}=0.0936 \\
& \bar{I}_{B}=\text { Const. }+w_{1} \times \hat{p}_{B_{1}}+w_{2} \times \hat{p}_{B_{2}}=-0.3269 \\
& \bar{I}=\left(n_{A} \bar{I}_{A}+n_{B} \bar{I}_{B}\right) /\left(n_{A}+n_{B}\right)=-0.1517
\end{aligned}
$$

Theoretically, the value of $\bar{I}$ is expected to be equal to 0 by (2.8), but the actual value of $\bar{I}$ is slightly different from 0 by computational error.

So we have the next discrimination rule by neglecting the approximation error Min. $S$

Actual value of $I>-0.1517$

$$
\text { ........case } Y \epsilon A \text { is predicted. }
$$

Actual value of $I<-0.1517$

$$
\text { .........case } Y \epsilon B \text { is predicted. }
$$

In Table 5, the precentage of success and skill score are computed in the usual way as follows:

For example, in the case of an approximate

\begin{tabular}{|c|c|c|c|}
\hline Actual Pred & A & B & Total \\
\hline A & 33 & 17 & 50 \\
\hline B & 22 & 48 & 70 \\
\hline Total & 55 & 65 & 120 \\
\hline
\end{tabular}
linear discriminator, percentage of success

$$
\begin{gathered}
=\frac{n_{11}+n_{22}}{n}=\frac{33+48}{120}=0.68 \\
\text { skill score }=\frac{n\left(n_{11}+n_{22}\right)-\left(n_{A}^{2}+n_{B}^{2}\right)}{n^{2}-\left(n_{A}^{2}+n_{B}^{2}\right)}=0.31
\end{gathered}
$$

If the approximation error Min.S is to be taken into account, we have $\sqrt{\mathrm{Min} . \mathrm{S} / \mathbf{2}^{2}} \div 0.091$ as the mean standard error, so the range $(-0.1517+0.091,-0.1517-0.091)$ may be regarded as a kind of doubtful region.

Table 5. Contingency tables of verification in the prediction of rainfall at the Hokuriku district $(s=2)$.

(a) Exact discriminator

\begin{tabular}{c|c|c|c}
\hline Actual Pred & A & B & Total \\
\hline A & 34 & 16 & 50 \\
B & 21 & 49 & 70 \\
\hline Total & 55 & 65 & 120 \\
\hline
\end{tabular}

Percentage of success $=0.70$

Skill score $\quad=0.34$

(b) Approximate discriminator

Percentage of success $=0.68$

Skill score $\quad=0.31$

(b) Case 2. $s=3$

To predict $Y$ by use of the three predictors $X_{1}$, $X_{2}$ and $X_{3}$ similarly to Case 1 , the next computations are performed:

$$
\begin{gathered}
\text { const. }=\sum_{i=1}^{3}\left(\hat{q}_{A i} / \widehat{q}_{B i}\right)=-1.5976 \\
\tilde{\rho}_{123(A)}=\frac{\widetilde{E}_{A}\left(X_{1}-\hat{p}_{A_{1}}\right)\left(X_{2}-\hat{p}_{A_{2}}\right)\left(X_{3}-\hat{p}_{A_{3}}\right)}{\sqrt{\hat{p}_{A_{1}} \hat{q}_{A_{1}} \hat{p}_{A_{2}} \hat{q}_{A_{2}} \hat{p}_{A_{3}} \hat{q}_{A_{3}}}} \\
\tilde{\rho}_{123(B)}=\frac{\widetilde{E}_{B}\left(X_{1}-\hat{p}_{B_{1}}\right)\left(X_{2}-\hat{p}_{B_{2}}\right)\left(X_{3}-\hat{p}_{B_{3}}\right)}{\sqrt{\hat{p}_{B_{1}} \hat{q}_{B_{1}} \hat{p}_{B_{2}} \hat{q}_{B_{2}} \hat{p}_{B_{3}} \hat{q}_{B_{3}}}} \\
\fallingdotseq 0016 \\
f(1,1,1)=\log \left\{1+\tilde{\rho}_{12(A)} \sqrt{\hat{q}_{A_{1}} \hat{q}_{A_{2}} / \hat{p}_{A_{1}} \hat{p}_{A_{2}}}\right.
\end{gathered}
$$




$$
\begin{aligned}
& +\tilde{\rho}_{13(A)} \sqrt{\hat{q}_{A_{1}} \hat{q}_{A_{3}} / \hat{p}_{A_{1}} \hat{p}_{A_{3}}}+\tilde{\rho}_{23(A)} \sqrt{\hat{q}_{A_{2}} \hat{q}_{A_{3}} / \hat{p}_{A_{2}} \hat{p}_{A_{3}}} \\
& \left.+\tilde{\rho}_{123(A)} \sqrt{\hat{q}_{A_{1}} \hat{q}_{A_{2}} \hat{q}_{A_{3}} / \hat{p}_{A_{1}} \hat{p}_{A_{2}} \hat{p}_{A_{3}}}\right\} \\
& -\log \left\{1+\tilde{\rho}_{12(B)} \sqrt{\hat{q}_{B_{1}} \hat{q}_{B_{2}} / \hat{p}_{B_{1}}{\hat{p_{B}}}_{B_{2}}}\right. \\
& +\tilde{\rho}_{13(B)} \sqrt{\hat{q}_{B_{1}} \hat{q}_{B_{3} / \hat{p}_{B_{1}} \hat{p}_{B_{3}}}}+\tilde{\rho}_{23(B)} \sqrt{\hat{q}_{B_{2}} \hat{q}_{B_{3}} / \hat{p}_{B_{2}} \hat{p}_{B_{3}}} \\
& \left.+\tilde{\rho}_{123(B)} \sqrt{\hat{q}_{B_{1}} \hat{q}_{B_{2}} \hat{q}_{B_{3}} / \hat{p}_{B_{1}} \hat{p}_{B_{2}} \hat{p}_{B_{3}}}\right\} \fallingdotseq-0.4656 \\
& \text { similarly } \\
& f(1,1,0)=0.0094, \quad f(1,0,1)=-0.2724 \\
& f(0,1,1)=-0.4303, f(1,0,0)=-0.3038 \\
& f(0,1,0)=0.1149, \quad f(0,0,1)=0.8180 \\
& f(0,0,0)=0.3165
\end{aligned}
$$

and so we have

$$
\begin{array}{ll}
a_{1}=-0.2483, & a_{2}=-0.1159, \quad a_{3}=0.0947 \\
w_{1}=0.6730, & w_{2}=0.9255,
\end{array}
$$

Min. $S=0.1048, \bar{I}_{A}=0.2361, \overline{I_{B}}=-0.5410$

$\bar{I}=-0.2172$

The next discrimination rule can be approximately induced:

Actual $I>-0.2172 \cdots \cdots \cdots$ case $Y \epsilon A$ is predicted. Actual $I<-0.2172 \cdots \cdots \cdots$. case $Y \in B$ is predicted.

As in Case $1(s=2)$, the correspondence between discriminated result and actual case is given by Table 6, and the percentages of success and skill score are obtained in the same way.

Table 6. Contingency tables of verification in the prediction of rainfall at the Hokuriku district $(s=3)$.

(a) Exact discriminator

\begin{tabular}{c|c|c|c} 
Actual Pred & A & B & Total \\
\hline A & 37 & 13 & 50 \\
B & 19 & 51 & 70 \\
\hline Total & 56 & 64 & 120 \\
\hline
\end{tabular}

Percentage of success $=0.73$

Skill score

$$
=042
$$

(b) Approximate discriminator

\begin{tabular}{c|c|c|c} 
Actual Pred & A & B & Total \\
\hline A & 36 & 14 & 50 \\
B & 19 & 51 & 70 \\
\hline Total & 55 & 65 & 120 \\
\hline
\end{tabular}

Percentage of success $=0.72$

Skill score $=0.40$
Certainly, the success rate of prediction is larger in Case 2 than in Case 1 for the dependent data, as is expected from a consideration of predictors, and there is almost no difference between exact discriminator and approximate linear discriminator so long as the success rate of prediction is concerned. However, it is not always sufficient in the present forecast routine, and numerous effective predictors must be taken up in this way to attain to practical accuracy.

(ii) Discrimination of two modes of snowslide by means of various vegetational and geomorphological conditions.

Snowslides can be conventionally classiified into avalanche (Nadare) and snow-crack (Yukiwareme).

Relevant data have been obtained by graphical representation of the aerial photographical observation projected in 1968 , by the Meteorological (Counter measure) Committee of Express Highway Research Foundation of Japan and they are used in this paper with the permission of the chief of the above Committee.

To have a discriminator for the snowslide, the following notations and assumptions are conventionally adopted:

To make it available for actual use, the decision rule concerning the occurrence or non-occurrence of snowslide at any given slope must of course be established so as to make possible a preventive measure, but such data have not been obtained so far.

Event $Y \epsilon A=$ avalanche occurred on a mountain slope.

Event $Y \epsilon B=$ snow crack occurred in part of a mountain slope.

The total number of factors is equal to 63 and the sample size $\mathrm{N}=516$, which were measured by $\mathrm{T}$. Ogawa and the computation was carried out by $\mathrm{M}$. Hirose using these possible factors.

However, in this paper, ten factors are selected from among them as empirically effective ones suggested by several experts, to show only a comutational example of theoretical result mentioned in Section 3.

Explanation of the ten selected factors (predictors) and their category definitions are given in Table 7.

Sample sizes are as follows:

Total sample size $n=n_{A}+n_{B}=516$

Sample size of case $Y \epsilon A: n_{A}=123$

Sample size of case $Y \epsilon B: n_{B}=393$ 
Table 7. Explanation of the selected geomorphological and vegetational factors and each category.

\begin{tabular}{|c|c|c|c|}
\hline \multirow{2}{*}{$\begin{array}{l}\text { Factor No. } \\
\text { (Predictor) }\end{array}$} & \multirow{2}{*}{ Explanation of Factor } & \multicolumn{2}{|r|}{ Category } \\
\hline & & size $(k i)$ & Categorical classification \\
\hline$X_{1}$ & Outline of slope & 6 & $\begin{array}{lll}C_{11}=\nabla & C_{12}=\square & C_{13}=\triangle \\
C_{14}=\square & C_{15}=\square & C_{16}=\square\end{array}$ \\
\hline$X_{2}$ & Longitudinal profile of slope & 4 & $\begin{array}{l}C_{21}=\text { flat }, \quad C_{22}=\text { concave } \\
C_{23}=\text { convex }, C_{24}=\text { mixed type uneven }\end{array}$ \\
\hline$X_{3}$ & Traverse profile of slope & 4 & $\begin{array}{ll}C_{31}=\text { flat }, & C_{32}=\text { concave } \\
C_{33}=\text { convex }, & C_{34}=\text { mixed type uneven }\end{array}$ \\
\hline$X_{4}$ & Dimensions of slope & 3 & $\begin{array}{l}C_{41}=\text { narrow }, C_{42}=\text { moderate } \\
C_{43}=\text { wide }\end{array}$ \\
\hline$X_{5}$ & Snow-cover & 3 & $\begin{array}{l}C_{51}=\text { light }, \quad C_{52}=\text { moderate } \\
C_{53}=\text { heavy }\end{array}$ \\
\hline$X_{6}$ & $\begin{array}{l}\text { Wind direction inferred from } \\
\text { Cornice }\end{array}$ & 4 & $\begin{array}{ll}C_{61}=\text { unknown }, & C_{62}=\text { upward } \\
C_{63}=\text { downward }, & C_{64}=\text { sideward }\end{array}$ \\
\hline$X_{7}$ & $\begin{array}{l}\text { Kind of forest trees } \\
\text { (Upper part of slope) }\end{array}$ & 3 & $\begin{array}{l}C_{71}=\text { broad leaf }, C_{72}=\text { needle leaf } \\
C_{73}=\text { mixed }\end{array}$ \\
\hline$X_{8}$ & Height of forest trees & 2 & $C_{81}=$ lower,$\quad C_{82}=$ higher \\
\hline$X_{9}$ & Density of forest trees & 4 & $\begin{array}{l}C_{91}=\text { sparse }, \quad C_{92}, C_{93}=\text { intermediate } \\
C_{94}=\text { dense }\end{array}$ \\
\hline$X_{10}$ & $\begin{array}{l}\text { Land-surface condition or } \\
\text { vegetation }\end{array}$ & 5 & $\begin{array}{l}C_{101}=\text { nakedrocks }, \quad C_{102}=\text { grassland } \\
C_{103}=\text { intermediate } \quad C_{104}=\text { shrub } \\
C_{105}=\text { forest (high) trees }\end{array}$ \\
\hline
\end{tabular}

Table 8. Quantification of each category for $X_{1}, X_{2}, \cdots \cdots, X_{10}$.

\begin{tabular}{|c|c|c|c|c|c|}
\hline Factor & Category & Quatification & Factor & Category & Quatification \\
\hline \multirow[b]{2}{*}{$X_{1}$} & $\begin{array}{l}C_{11} \\
C_{12}\end{array}$ & $\begin{array}{l}0.00 \\
1.38\end{array}$ & $X_{3}$ & $\begin{array}{l}C_{33} \\
C_{34}\end{array}$ & $\begin{array}{r}2.54 \\
-2.38\end{array}$ \\
\hline & $\begin{array}{l}C_{13} \\
C_{14} \\
C_{15} \\
C_{16}\end{array}$ & $\begin{array}{l}-0.01 \\
-0.22 \\
-0.51 \\
-0.01\end{array}$ & $X_{4}$ & $\begin{array}{l}C_{41} \\
C_{42} \\
C_{43} \\
\end{array}$ & $\begin{array}{r}0.17 \\
-0.33 \\
0.30\end{array}$ \\
\hline \multirow[t]{2}{*}{$X_{2}$} & $\begin{array}{l}C_{21} \\
C_{22} \\
C_{23}\end{array}$ & $\begin{array}{r}0.00 \\
0.96 \\
-0.49\end{array}$ & $X_{5}$ & $\begin{array}{l}C_{51} \\
C_{52} \\
C_{53} \\
\end{array}$ & $\begin{array}{r}0.00 \\
-5.46 \\
1.19 \\
\end{array}$ \\
\hline & $C_{24}$ & -0.20 & \multirow[b]{2}{*}{$X_{6}$} & \multirow{2}{*}{$\begin{array}{l}C_{61} \\
C_{62} \\
C_{63}\end{array}$} & -1.45 \\
\hline$X_{3}$ & $\begin{array}{l}C_{31} \\
C_{32}\end{array}$ & $\begin{array}{l}0.05 \\
0.03\end{array}$ & & & $\begin{array}{l}0.78 \\
0.48\end{array}$ \\
\hline
\end{tabular}




\begin{tabular}{c|c|c||c|c|c}
\hline Factor & Category & Quantification & Factor & Category & Quantification \\
\hline$X_{6}$ & $C_{64}$ & 1.89 & $X_{9}$ & $C_{93}$ & -0.34 \\
\hline \multirow{3}{*}{$X_{7}$} & $C_{71}$ & 0.73 & & $C_{9 \cdot 1}$ & 0.56 \\
& $C_{72}$ & -0.81 & & $C_{101}$ & 0.60 \\
& $C_{73}$ & -0.12 & & $C_{102}$ & 2.54 \\
$X_{8}$ & $C_{81}$ & 0.73 & $X_{10}$ & $C_{103}$ & -0.74 \\
& $C_{82}$ & -0.89 & & $C_{104}$ & -1.61 \\
\hline$X_{9}$ & $C_{91}$ & 0.73 & & $C_{105}$ & 0.16 \\
& $C_{12}$ & -0.64 & & & \\
\hline
\end{tabular}

Table 9. Statistical computations necessary to constitute discriminator $I$ for snowslide.

(a) Sample mean value

\begin{tabular}{c|cccccccccc}
\hline$i$ & 1 & 2 & 3 & 4 & 5 & 6 & 7 & 8 & 9 & 10 \\
\hline $\bar{X}_{A i}$ & $5.33^{-2}$ & $4.15^{-4}$ & $2.25^{-2}$ & $9.37^{-3}$ & $7.96^{-1}$ & $2.32^{-1}$ & $9.38^{-2}$ & $8.05^{-2}$ & $1.08^{-1}$ & $1.38^{-1}$ \\
\hline $\bar{X}_{B i}$ & $-1.70^{-1}$ & $-1.33^{-3}$ & $-7.18^{-2}$ & $-2.99^{-2}$ & $-2.54^{-1}$ & $-7.40^{-1}$ & $-3.00^{-1}$ & $-2.57^{-1}$ & $-3.46^{-1}$ & $-4.42^{-1}$ \\
\hline
\end{tabular}

(b) Sample variance and covariance matrix

\begin{tabular}{|c|c|c|c|c|c|c|c|c|c|c|}
\hline$j{ }^{i}$ & 1 & 2 & 3 & 4 & 5 & 6 & 7 & 8 & 9 & 10 \\
\hline 1 & $6.50^{4}$ & $6.47^{5}$ & $5.23^{5}$ & $4.97^{4}$ & 4. $26^{5}$ & 2. $42^{5}$ & $4.91^{4}$ & $-7.88^{2}$ & $-6.10^{2}$ & 4. $24^{1}$ \\
\hline 2 & & 6. $29^{6}$ & $6.30^{6}$ & $6.02^{5}$ & $5.26^{6}$ & $2.46^{6}$ & 5. $24^{5}$ & $-4.36^{4}$ & $-1.16^{4}$ & 7. $74^{2}$ \\
\hline 3 & & & 6. $39^{6}$ & $5.03^{5}$ & $4.85^{6}$ & 2. $20^{6}$ & 5. $07^{5}$ & $-5.19^{4}$ & $-1.44^{4}$ & $2.18^{3}$ \\
\hline 4 & & & & 1. $32^{5}$ & $4.65^{5}$ & $2.18^{5}$ & 4. $55^{4}$ & $-4.15^{3}$ & $-4.80^{2}$ & $-3.27^{2}$ \\
\hline 5 & & & & & 5. $30^{6}$ & $1.17^{6}$ & 4. $18^{5}$ & $-2.06^{4}$ & $-6.31^{3}$ & $2.20^{3}$ \\
\hline 6 & & & & & & 2. $44^{6}$ & $2.26^{5}$ & $-2.60^{4}$ & $-1.10^{4}$ & $-1.19^{3}$ \\
\hline 7 & & & & & & & 4. $92^{4}$ & $-3.41^{3}$ & $-9.55^{2}$ & $1.95^{2}$ \\
\hline 8 & & & & & & & & 5. $00^{3}$ & 1. $62^{2}$ & $-3.08^{1}$ \\
\hline 9 & & & & & & & & & 5. $91^{2}$ & 1. $29^{2}$ \\
\hline 10 & & & & & & & & & & 7. $15^{2}$ \\
\hline
\end{tabular}

(c) Discriminator coefficient

\begin{tabular}{|c|c|c|c|c|c|c|c|c|c|c|}
\hline$i$ & 1 & 2 & 3 & 4 & 5 & 6 & 7 & 8 & 9 & 10 \\
\hline$w_{t}$ & $8.45^{-3}$ & $2.78^{-4}$ & $-7.89^{-4}$ & 3. $82^{-4}$ & $-3.13^{-4}$ & $-4.26^{-3}$ & $2.04^{-2}$ & $-1.38^{-2}$ & $-9.14^{-2}$ & $-4.75^{-3}$ \\
\hline
\end{tabular}

Remark: For example, $5,33^{-2},-1.70^{-1}, 6.50^{4}$ and $8.45^{-3}$ are $5.33 \times 10^{-2},-1.70 \times 10^{-1}, 6.50 \times 10^{4}$ and $8.45 \times 10^{-3}$ respectively. 
Table 10. Contingency tables of verification (Snowside).

(a) $s=10, X$

\begin{tabular}{c|r|r|r}
\hline Actual Pred & A & B & Total \\
\hline A & 300 & 93 & 393 \\
B & 10 & 113 & 123 \\
\hline Total & 310 & 206 & 516 \\
\hline
\end{tabular}

Percentage of success $=81 \%$

Skill score $\quad=0.34$

(b) $s=52, X$

\begin{tabular}{c|r|r|c}
\hline Actual Pred & A & B & Total \\
\hline A & 341 & 52 & 393 \\
B & 6 & 117 & 123 \\
\hline Total & 347 & 169 & 516 \\
\hline
\end{tabular}

Percentage of success $=90 \%$

Skill score $\quad=0.58$

Quantified results of categorical variables are given in Table 8, and the necessary statistical values are summarized in Table 9. As is shown in Table $9, X_{7}$ is the most effective factor for our discrimination, and discriminator $I$ can be expressed as

$$
\begin{aligned}
I= & 0.00845 X_{1}+0.00028 X_{2}-0.00079 X_{3} \\
& +0.00038 X_{4}-0.00031 X_{5}-0.00426 X_{6} \\
& +0.0204 X_{7}-0.0138 X_{8}-0.0914 X_{9} \\
& -0.00475 X_{10}
\end{aligned}
$$

by use of Table 9 . (c).

In this case, the discrimination rule is determin ed as in the following:

Actual values of $I>0 \ldots \ldots$ case $Y \epsilon A$ is predicted. Actual values of $I<0 \cdots \cdots$ case $Y \epsilon B$ is predicted.

Verification of the results of snowslide discrimination can be performed based on the above discrimination rule for dependent data, and a part of the summarized results is shown in Table 10.

As is seen in Table 10, the result of snowslide discrimination is not always satisfactory for practical use, since the sufficiently effective predictors may not be always selected from the possible factors of size 63 in this example.

However, judging from the result shown in Table 10 (b), it will be possible to obtain a practically useful discrimination procedure through a screening method similar to the one that R.G.
Miller proposed for the construction of quantitative linear discriminant function.

\section{Concluding remark}

Two statistical procedures of discrimination have been given in this paper by use of several qualitative or categorical variables (predictors) with their applications to meteorological problems.

Speaking generally of the essential points, in order to predict either of two mutually exclusive events, each variable taken up as an effective predictor has to be specified as a simple qualitative variable having a tendency to be equal to 1 when it is more snitable for one case (Event $Y \epsilon A$ ) and to (equal to) 0 for the other case (Event $Y \epsilon B$ ) So, there is no need to have three or more values or categories for each variable, so long as the two events only are considered.

Consequently, discriminator I based on the likelihood ratio of two multi-variate binomial distributions has a sufficient generality for discrimination of the two exclusive cases of predictand, and we may consider the sequential approach to constitute such discriminator in a stepwise manner for discrimination of the three or more exclusive cases in view of fundamental standpoint as follows:

For example, let the possible cases of $Y$ be $A_{1}$, $A_{2}, \cdots \cdots \cdots, A_{k}$ (they are assumed generally to be mutually exclusive and exhaustive), then we can have the following stepwise discriminators:

The lst discriminator for the one Event $Y \epsilon A$, and the other Event $Y \epsilon \sum_{i=2}^{k} A_{i}$

The 2nd discriminator for the one Event $Y \epsilon A_{2}$ and the other Event $Y \epsilon \sum_{i=3}^{k} A_{i}$

The $k-1$-th discriminator for the one Event $Y \in A_{k-1}$ and the other last Event $Y \epsilon A_{k}$

And for each stepwise discriminator, we can devise so as to take predictor variables having tendencies to equal to 1 when they are suitable for the one Event and to 0 for the other exclusive Event.

The procedure combining the categorical variables of the simultaneous equation (3.7) to have the discrimination coefficients whose methodological originality is to be ascribed to T. Hayashi and S. chino, was first applied in this paper to meteorological problems in a slightly different way from the usual method. 


\section{Ackowledgement}

The author wishes to express his sincere thanks to the staff members of the Forecast Research Laboratory in M.R.I. for their valuable discussions and especially to Mr. M. Hirose for his programming and computation throughout this. $\mathrm{He}$ is also indebted to Miss J. Hashiba for her unstinted assistance. The author would like to dedicate this paper to Prof. E. Fukui in commemoration of his retirement from Tokyo Educational University.

\section{Appendix}

[Proof of Theorem referred to in P. 3]

For this linear approximation, the residual sum of square error can be formally written as

$$
S=\sum_{x_{1}=0}^{1} \sum_{x_{3}=0}^{1} \cdots \sum_{x_{s}=0}^{1}\left\{f\left(x_{1}, x_{2}, \cdots, x_{s}\right)-\sum_{i=1}^{s} a_{i} x_{i}\right\}^{2}
$$

and $S$ is a function of $\left(a_{1}, a_{2}, \cdots, a_{s}\right)$

In order to minimize $S$ in respect to $a_{1}, a_{2}, \cdots$, $a_{s}$, the necessary conditions

$$
\partial S / \partial a_{i}=0 \quad(i=1,2, \cdots, s)
$$

are explicitly shown by the next simultaneous linear equation:

$$
\begin{aligned}
& 2^{s-1} a_{1}+2^{s-2}\left(a_{2}+a_{3}+\cdots+a_{s}\right) \\
&= \sum_{x_{2}=0}^{1} \sum_{x_{3}=0}^{1} \cdots \cdots \sum_{x_{s}=0}^{1} f\left(1, x_{2}, x_{3}, \cdots, x_{s}\right) \\
& 2^{s-1} a_{2}+2^{s-2}\left(a_{1}+a_{3}+\cdots+a_{s}\right) \\
&=\sum_{x_{1}=0}^{1} \sum_{x_{3}=0}^{1} \cdots \cdots \sum_{x_{s}=0}^{1} f\left(x_{1}, 1, x_{3}, \cdots, x_{s}\right) \\
& \cdots \cdots \cdots \cdots \cdots \cdots \cdots \\
& 2^{s-1} a_{s}+2^{s-2}\left(a_{1}+a_{2}+\cdots+a_{s-1}\right) \\
&=\sum_{x_{1}=0}^{1} \sum_{x_{2}=0}^{1} \cdots \cdots \sum_{x_{s-1}=0}^{1} f\left(x_{1}, x_{2}, \cdots, x_{s-1}, 1\right)
\end{aligned}
$$

Let the notations $g_{1}, g_{2}, \cdots, g_{s}$ be put as the first, second, and the $s$-th column in the right multiple summations respectively, then the solution of this simultaneous equation can be easily written as

$$
\begin{array}{r}
a_{1}=2^{2-s}\left\{g_{1}-\sum_{i=1}^{s} g_{i} /(s+1)\right\}, \\
a_{2}=2^{2-s}\left\{g_{2}-\sum_{i=1}^{s} g_{i} /(s+1)\right\}, \\
\ldots \ldots \ldots \ldots \ldots \ldots \ldots \ldots \ldots \ldots \ldots \ldots \\
a_{s}=2^{2-s}\left\{g_{s}-\sum_{i=1}^{s} g_{i} /(s+1)\right\}
\end{array}
$$

Consequently, the weight coefficients $w_{i}(i=1$, $\cdots, s)$ will be shown from (2.8) as follows:

$$
\begin{aligned}
w_{i} & =\log \left(p_{A i} q_{B i} / p_{B i} q_{A i}\right)+a_{i} \\
& =\log \left(p_{A i} q_{B i} / p_{B i} q_{A i}\right)+2^{2-s}\left\{g_{i}-\sum_{i=1}^{s} g_{i} /(s+1)\right\}
\end{aligned}
$$

In the next place, let Min. $S$ be the minimum value of $S$ obtained by substituting these results of $a_{1}, a_{2}, \cdots, a_{s}$, then we have

$$
\text { Min. } \begin{aligned}
S & =\sum_{x_{1}=0}^{1} \sum_{x_{2}=0}^{1} \cdots \sum_{x_{s}=0}^{1} f\left(x_{1}, x_{2}, \cdots, x_{s}\right)^{2}-2 \sum_{i=1}^{s} a_{i} g_{i} \\
& +2^{s-1}\left(\sum_{i=0}^{s} a_{i}{ }^{2}+\sum_{i=0}^{s-1} a_{i} a_{i+1}\right) \\
& =\sum_{x_{1}=0}^{1} \sum_{x_{2}=0}^{1} \cdots \sum_{x s=0}^{1} f\left(x_{1}, x_{2}, \cdots, x_{s}\right)^{2} \\
& -2^{3-s}\left\{\sum_{i=1}^{s} g_{i}{ }^{2}-\sum_{i=1}^{s} g_{i}{ }^{2} /(s+1)\right\} \\
& +2^{2-s}\left\{\left(\sum_{i=1}^{s} g_{i}\right)^{2} /(s+1)^{2}\right. \\
& +\sum_{i=1}^{s} g_{i}^{2}-2\left(\sum_{i=1}^{s} g_{i}\right)^{2} /(s+1) \\
& \left.+s\left(\sum_{i=1}^{s} g_{i}\right)^{2} /(s+1)^{2}\right\} \\
& =\sum_{x_{1}=0}^{1} \sum_{x_{2}=0}^{1} \ldots \sum_{x s=0}^{1} f\left(x_{1}, x_{2}, \cdots, x_{s}\right)^{2} \\
& +2^{2-s}\left\{\left(\sum_{i=1}^{s} g_{i}\right)^{2} /(s+1)-\sum_{i=1}^{s} g_{i}^{2}\right\}
\end{aligned}
$$

\section{References}

Chino, S., 1963: The relation between correlation ratio and success rate in the classification by the quantification method. Proc. Inst. Stat. Math., 11,7-24, (in Japanese)

Dwyer, P.S., 1951: Linear computations. John Wiley and Sons.

Hayashi, C., 1952: Fundamental concept of the theory of quantification and prediction. Proc. Inst. Stat. Math. 7, 43-64. (in Japanese)

Householder, A.S., 1953: Principles of numerical analysis. McGraw-Hill, New York.

Krishnamoorthy, A. S., 1951: Multi-variate binomial and Poisson distribution. Sankhya, 11, 117-124.

Maruyasu, T. and his collaborators, 1964: A study on landslide at the neighbour region of Yoshino river by aerial photographs.

Sokuryo 6, 8-13 (in Japanese)

Miller, R.G., 1962: Statistical prediction by discriminant analysis. Met. Monogr., 4, 25. Amer. 
Met. Soc.

Suzuki, E., 1964: Categorical prediction schemes of rainfall types by discriminant analysis. Papers in Met. and Geophys. 15, 119-160.
Suzuki, E., 1966: Correlation analysis containing discrete variables used in certain meteorological problems. Papers in Met. and Geophys. 17, 10-30.

\section{定性的な変量による統計的判別理論とその気象への応用}

鈴木栄一

気象研究所

これまでの判別関数による気象予測は R. G. Miller らと筆者により研究されてきたが，それは数值として得られ る連続変量の総合化したものであった。ここでは大雨や大雪, なだれなどの発生有無を事前に予測する場合, その手 がかりとなる因子が定性的な变量（たとえば下層ジェットや前線の有無, 地形, 植生形態を示すもので数值として得 られていない変量）である場合の判別方式を開発した。

まず筆者らによって定義された多变量 2 項分布の一般式から出発し，尤度比の対数を線形近似によって現場でも容 易に利用できる形になおし, 次に統計的数量化理論を気象予測の目的に合うょう定式化して判別予報式を導びき, こ

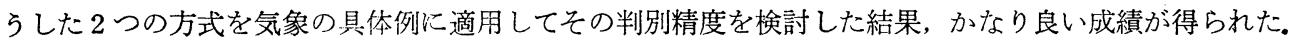

